\title{
Automatic Threshold on Current based Anti-pinch Mechanism for Power Windows
}

\author{
Wahyu Kunto Wibowo ${ }^{1}$, Seok-Kwon Jeong ${ }^{2}$, Sam-Sang You ${ }^{3}$, Jaemin Lee ${ }^{4}$ \\ ${ }^{1}$ Department of Electrical Engineering, Universitas Pertamina, Indonesia \\ ${ }^{2}$ Department of Referigeration and Air Conditioning, Pukyong National University, South Korea \\ ${ }^{3}$ Division of Mechanical Engineering, Korea Maritime and Ocean University, South Korea \\ ${ }^{4}$ Department of Interdisciplinary of Mechatronics Engineering, Pukyong National University, South Korea
}

\begin{tabular}{l}
\hline \hline Article Info \\
\hline Article history: \\
Received Jun 22, 2017 \\
Revised Mar 11, 2018 \\
Accepted Apr 10, 2018
\end{tabular}

\section{Keyword:}

Anti-pinch

Automatic threshold

Automotive power window

Contact method

Electric current information

FMVSS 118 regulation

\begin{abstract}
In this paper, the automotive power window has been integrated with an advanced safety mechanism called anti-pinch system for good protection. Based on a contact method, a new safety mechanism using a low-cost technology has been proposed to set a threshold value as a limit to decide the pinch condition, or automatic threshold method. The electric current information is easily detected by using current sensor installed on a motor driver without incorporating extra device. Then the pinch condition is quickly reflected and calculated by using current information in the system. Since the automatic threshold is decided by analyzing system behavior in advance, the optimal calculation can be guaranteed and then applied it on the safety mechanism in a cost-effective manner. Through extensive experimental tests, the squeezing forces of the proposed anti-pinch system have been verified to satisfy requirements of the FMVSS 118 regulations.
\end{abstract}

Copyright $\odot 2018$ Institute of Advanced Engineering and Science. All rights reserved.

\section{Corresponding Author:}

Wahyu Kunto Wibowo,

Department of Electrical Engineering,

Universitas Pertamina,

Komplek Universitas Pertamina, Jalan Teuku Nyak Arief, Simprug, Kebayoran Lama, Jakarta, Indonesia.

Email: wahyu.kw@universitaspertamina.ac.id

\section{INTRODUCTION}

An anti-pinch system is a vehicle safety mechanism on a power window that prevents drivers and passengers from fatal injury and death caused by human body stuck or pinched in the window area [1], [2]. When the power window detects obstacle intrusion on the specified area, the anti-pinch system will begin to work, and the motorized window will go down automatically. There are two types of mechanisms in which the anti-pinch system works: a non-contact method and a contact method. The non-contact system detects the position of the obstacle using sensors (for example, electromagnetic field sensor) to measure proximity distance of the obstacle that enters the sensor space-charge field before the window touches the obstacle. This approach is rarely used on power windows that incorporate anti-pinch system because it is a high cost of mechanism compared to the similar performance [3]. On the other hand, the contact method which senses obstacle intrusion on the window is generally based on the anti-pinch system. This scheme senses the obstacle by a direct touch between windows and obstacles that affects the DC motor behavior [4].

An anti-pinch technology has been proposed by processing the change in DC motor speed when the window glass touches the obstacle [5]-[7]. The speed information is obtained by installing number of Hall IC speed sensors near the rotor ring magnet of the DC motor. The output of the sensor is a signal period of the DC motor speed. When the window glass touches the obstacle, the period of the motor speed is longer than the normal period. By applying a threshold on the period, this period threshold for determining the pinch condition is set manually by the programmer. A contact method anti-pinch system has been suggested by 
utilizing the angular velocity of the DC motor and then estimating the torque rate from the angular velocity information to decide the pinch condition [8]-[11]. The torque rate is estimated by using Kalman filter and $H_{\infty}$ filter. Then the estimation is used to determine the threshold value and set manually afterwards. In addition, an anti-pinch system is suggested for a sunroof using fuzzy logic scheme [12], [13]. A membership function is made from relation between operation temperature and voltage to decide the pinch condition. The squeezing force can be obtained after comparing the membership function.

In the present study, the anti-pinch system is built by utilizing the electric current of the DC motor as the main information to decide the pinch condition. The current sensor is installed on DC motor driver. The key benefit of using current information is that the pinch condition reflects and detects quickly with good sensitivity. However, utilizing current information has some disadvantages, such as proneness to disturbances that can lead noises, which will make a false pinch alarm into the system. Moreover, the changes in DC motor parameters during the operation are also able to cause false pinch alarm. On the operational time, when the DC motor starts to work, the electric current generates heat or thermal energy on the DC motor. The heat from the DC motor affects the current information of the DC motor. If the manual threshold is applied to the system which is known to have these defects, the system with a low reliability will cause a lot of false pinch alarms.

In the present anti-pinch system, the automatic threshold is made and proposed as a new scheme for the current limit to decide the pinch condition. To realize this low-cost technique, the current characteristics are observed beforehand, and analyzed the optimal current threshold value to apply it into the anti-pinch mechanism. In the experimental tests, the best current threshold is should be acquired and fit the anti-pinch system. Finally, the squeezing force test is conducted on the proposed anti-pinch system, and the squeezing force is supposed to meet the FMVSS 118 safety standards.

\section{RESEARCH METHOD}

Europe and America have published rigorous regulations on the power windows for the automobile's safety: European regulation of DIRECTIVE 2000/4/EC,11 and American regulation of FMVSS 118-S5 [14], [15]. These regulations specify the standard limit on the maximum amount of squeezing force and the minimum safe reversal distances of the power windows which must be confirmed by an anti-pinch system for safety reasons. In the anti-pinch mechanism developed in this research, the electric current information is directly obtained by current sensor using Hall Effect, which attached on the DC motor driver. The regulations for improvement are imposed to make the automotive power windows safer and more comfortable for the drivers and the passengers. In this safety with convenience, America strengthens the vehicle safety standards applicable in the European region. Table 1 state the key features for DIRECTIVE 2000/4/EC and DOT FMVSS 118-S5 regulations. The squeezing force, reversal section, and reversal distances (in cases of \#1 and \#2) are the same for both regulations, but the test rod force deflection ratio and reversal distance in case of \#3 are different. The force deflection ratio is dependent on the size of the test rod itself. Two test rods are needed in FMVSS 118. Note that the FMVSS 118 regulation is more rigorous by using $65 \mathrm{~N} / \mathrm{mm}$ test rod.

Furthermore, the window area is divided into two work zones for the anti-pinch system; stop zone and reversal zone. The reversal zone on the window area is from $4 \sim 200 \mathrm{~mm}$ and other than this area is defined as the stop zone. The anti-pinch work zone is shown in Figure 1. Based on electric current, the antipinch system with contact method is suggested to satisfy FMVSS 118 regulation. The block diagram of the designed anti-pinch system is shown on Figure 2.

Table 1. Power window Regulations 10,11

\begin{tabular}{|c|c|c|}
\hline Item & DIRECTIVE 2000/4/EC & DOT FMVSS 118 \\
\hline Squeezing force & \multicolumn{2}{|c|}{$<100 \mathrm{~N}$} \\
\hline Reversal section & \multicolumn{2}{|c|}{$4-200 \mathrm{~mm}$} \\
\hline $\begin{array}{l}\text { Test rod force } \\
\text { deflection ratio }\end{array}$ & \multicolumn{2}{|r|}{$\begin{array}{l}\text { - } 65 \mathrm{~N} / \mathrm{mm}(\leq 25 \mathrm{~mm} \text { test rod }) \\
\bullet 20 \mathrm{~N} / \mathrm{mm}(>25 \mathrm{~mm} \text { test rod })\end{array}$} \\
\hline Reversal distances & \multicolumn{2}{|c|}{$\begin{array}{l}\text { 1. Same position when the window begins to close. } \\
\text { 2. A position that permits a semi-rigid cylindrical rod which have diameter } 200 \mathrm{~mm} \text {. } \\
\text { 3. A position at least } 50 \mathrm{~mm} \text { more } 3 \text {. A position at least } 125 \mathrm{~mm} \text { more open } \\
\text { open after the window start } \\
\text { reversing. }\end{array}$} \\
\hline
\end{tabular}




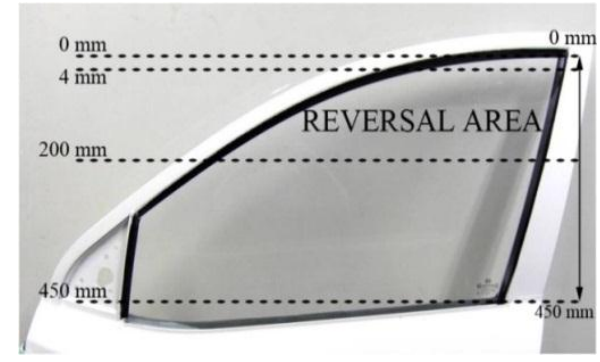

Figure 1. Anti-pinch system work zone on the window area for the safety regulation

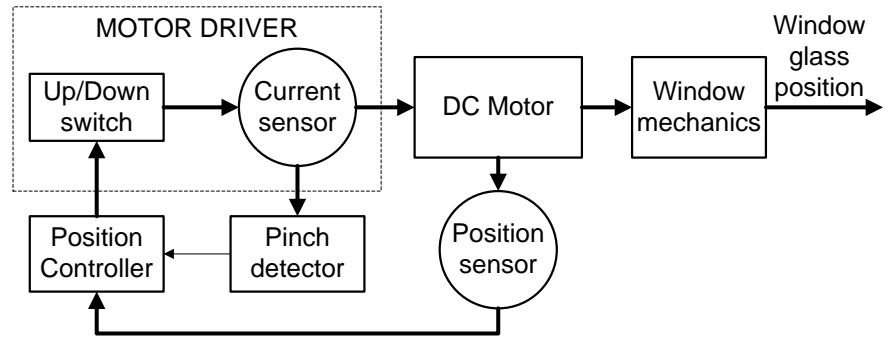

Figure 2. The schematic diagram of the electric current based anti-pinch system

The anti-pinch system is based on the current change of the DC motor that works as the power window actuator. If the obstacles are on the window area, and the window glass gets in contact with the obstacles, the DC motor will get load disturbance because the obstacle makes the DC motor current rise. This current change will be detected by the current sensor and if the current value is over the current threshold, it will be detected as a pinch condition. The automatic threshold method is proposed in this anti-pinch system. The position sensor is also used in the anti-pinch system as a supplementary sensor that checks the window glass position on the window frame. Based on the safety regulations, the position of the window glass on the frame is important to decide the danger zone and must contain a different response on the stop zone and the reversal zone. When the system detects a pinch condition, it will directly check the position of the window glass to decide in which position the pinch condition has occurred.

\subsection{DC motor current characteristics}

In this paper, the automatic threshold has been proposed to make a pinch decision in the anti-pinch system. Observing the current characteristics and analyzing the best calculation should be done before the automatic threshold is applied to the system. Figure 3 describes the block diagram for the complete power window model including DC motor as an actuator. The complete system can be divided into DC motor part and window mechanics part, which contains a set of mechanical gears and window glass.

The applied voltage $\mathrm{V}$ for the DC motor is $12 \mathrm{~V}$. The DC motor current value can be obtained from the signal generated by the current sensor. The DC motor current is the armature current $I_{a}$, and the pinch condition is obtained by processing this armature current. From the linear power window model in Figure 3, the total armature current $I_{a}$ of the DC motor for the anti-pinch system is obtained as follows:

$$
I_{a}(s)=\frac{V(s)(b+J s)+K_{e} T_{d}}{\left((b+J S)\left(R_{a}+L_{a} s\right)+K_{e} K_{t}\right)}
$$

From Figure 4, the DC motor current value is not consistent at all the time. From 1.5 to $3.3 \mathrm{~s}$, the window reaches the curvature zone of the window frame. During this time period, the friction between the window glass and the frame is changing when the system goes by, so the disturbance torque $T_{d}$ is rising, thus making the DC motor current rise. The anti-pinch system with automatic current threshold uses these DC motor current characteristics. The automatic current threshold should cover and work for all the time when the system operates. 


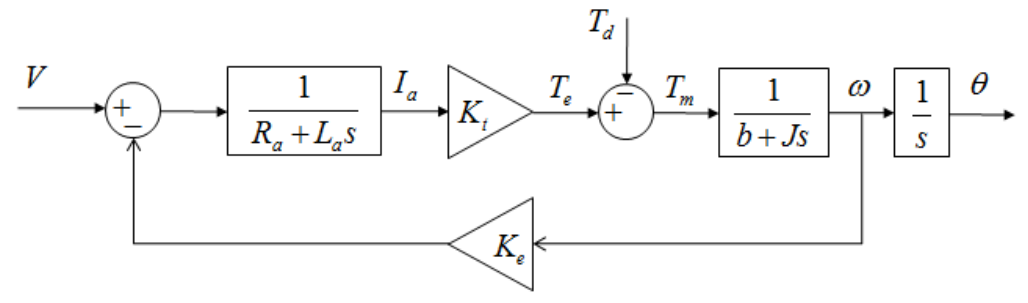

Figure 3. The block diagram of the power window model

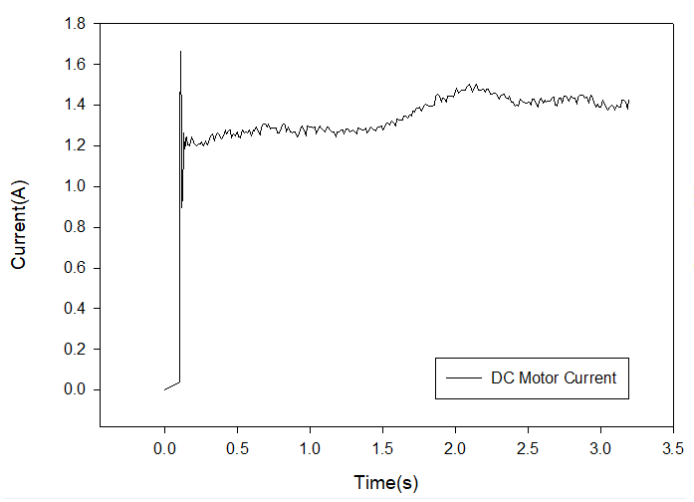

(a)

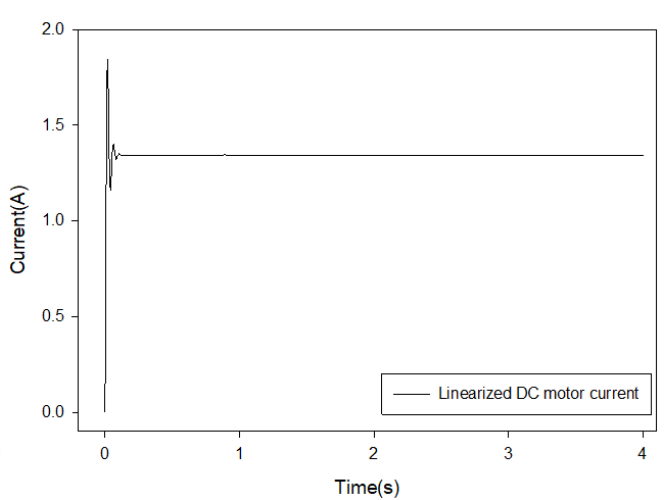

(b)

Figure 4. DC motor current characteristic (a) Actual, (b) Linearized model

\subsection{Pinch decision using automatic threshold method}

In a real system, the current amplitude value of the DC motor is always changing depending on the DC motor condition. The new DC motor current characteristics will be different from old DC motor characteristics. Also the operational timing will cause the DC motor current amplitude to change. By using automatic threshold method, the anti-pinch system is designed to overcome those problems. The pinch decision using the automatic threshold method is determined by analyzing the DC motor current characteristics on the power window system. Using system identification scheme, the DC motor current model can be predicted as follows:

$$
\frac{I(s)}{V(s)}=\frac{0.00025 s+0.1119}{0.0000416 s^{2}+0.004 s+1}
$$

where all the parameters of the system have already contained DC motor model, window mechanics, and the friction characteristics between the window glass and window frames.

Even though the system disturbance torque is changing, it will not affect the transient state of the current value. Widyan et al. [16] analyzed that the transient response on the DC motor will change significantly by the applied voltage type. In this research, it is found that the current value in the transient time has perfectly covered all the DC motor current value on the power window operation. Based on this analysis, the automatic threshold will be calculated using the sample data from the current values at the area of DC motor transient time. After the area of the calculation for the automatic threshold is defined, the current threshold (Ith) can be calculated from Equation (1) at transient time using the current value at transient time $\left(I_{t r}\right)$ by

$$
I_{t h}=0.97 \times I_{t r}
$$

where a reasonable automatic threshold level to detect the pinch condition is $97 \%$ of the maximum current of the transient time. This method can be acknowledged as a systematic way to solve the pinch detection rather than the manual threshold. 
Based on Equation (3) as a calculation for the automatic threshold, the power window response is shown in Figure 5. It is noted that the DC motor current will be rising when the pinch condition occurs on the system. If the current is over the threshold level, the anti-pinch system will be activated to make the window glass move downwards on the reversal zone, and then the window will stop in the stop zone.

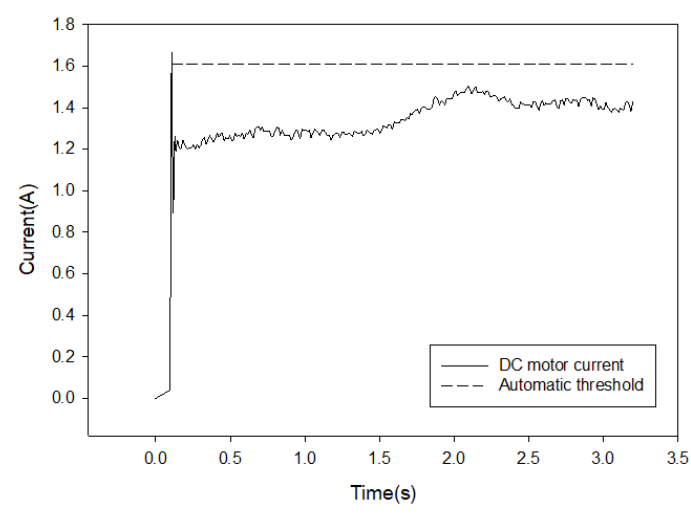

Figure 5. Automatic threshold applied to the power window anti-pinch system

\section{EXPERIMENTAL TESTS AND DISCUSSIONS}

In this experimental test, LabVIEW has been employed to control the DC motor driver and to calculate the DC motor current based on the sensor signals. In addition, LabVIEW is used to calculate the squeezing force of the anti-pinch system. Figure 6 describe the essential features of an experimental apparatus for power window system using LabVIEW. A load cell attached to the power window using a jig is used as a force sensor to detect the power window's squeezing force. As a test rod replacement, the test spring is attached on the load cell which has same force deflection ratio as FMVSS 118-S5 specification. Figure 7 shows the block diagram of the experimental system for power window anti-pinch system. To evaluate the pinch detection performance, the system is tested by using the test spring with standard deflection ratios of $20 \mathrm{~N} / \mathrm{mm}$ and $65 \mathrm{~N} / \mathrm{mm}$, as explained in the FMVSS 118 regulations.

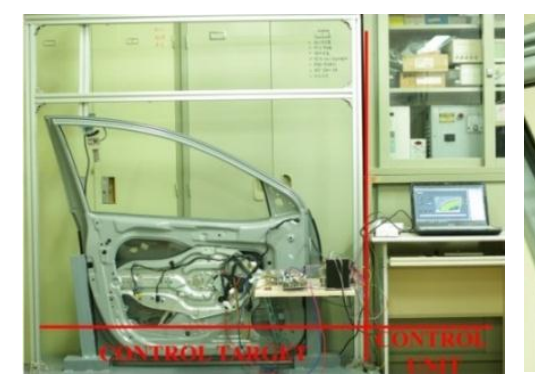

(a)

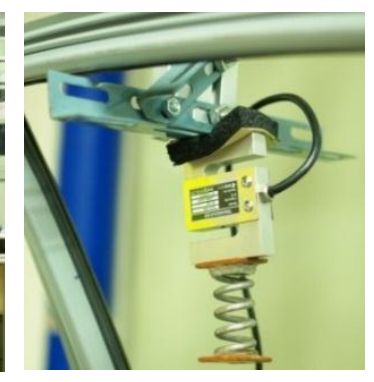

(b)

Figure 6. Experimental system (a) Full anti-pinch system, (b) Load cell

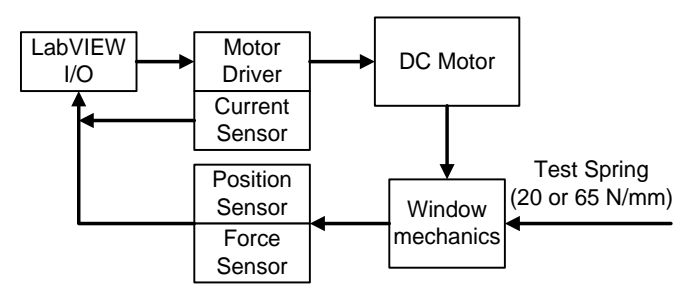

Figure 7. Block diagram of experimental system configuration 


\subsection{Pinch detection performance evaluation for $20 \mathrm{~N} / \mathrm{mm}$ test spring}

For each threshold method with manual and automatic, the pinch detection performances of the two methods are illustrated in Figure 8 with the current measurement. Under manual threshold case, the threshold value is set manually on 1.6 A. When the current is over the threshold level, the system will define the condition as the pinch detection. Under the automatic threshold, the system will calculate the threshold level by itself based on Equation (3). In this case, the pinch condition will be defined as same as the manual threshold.

As illustrated in Figures 8, when the pinched object is detected by the system, the current rises until its value reaches to the threshold value. After the current hits the threshold level, the system defines this as pinch detection, and the motor starts moving in reverse to make the window glass go back down. When the pinch detection is made, the force sensor which attached on the experimental system is working to measure the squeezing force. Table 2 shows the squeezing force results for both methods.

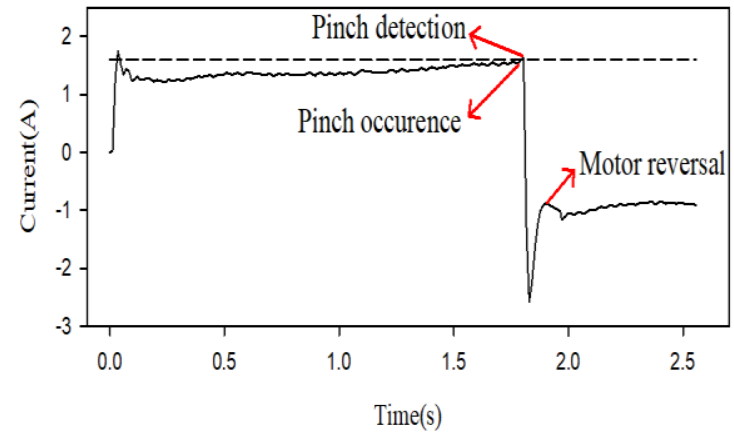

(a)

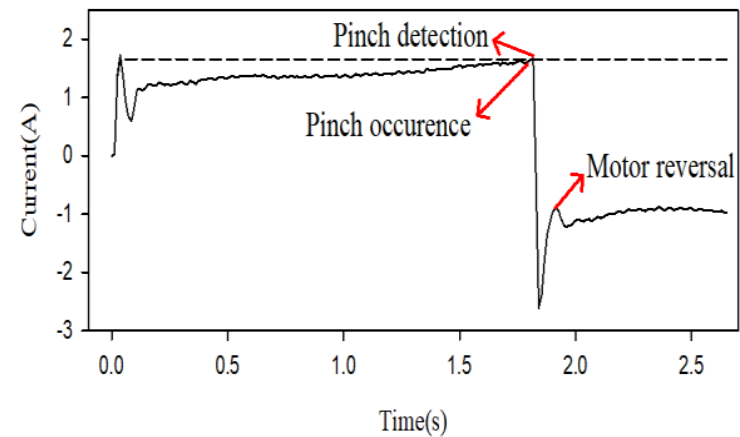

(b)

Figure 8. Pinch detection resuts using 20 N/mm test spring (a) Manual threshold, (b) Automatic threshold

Table 2. Squeezing Force Results for $20 \mathrm{~N} / \mathrm{mm}$ Test Spring

\begin{tabular}{ccccc}
\hline \multirow{2}{*}{ Test \# } & \multicolumn{2}{c}{ Manual threshold } & \multicolumn{2}{c}{ Automatic threshold } \\
& $I_{\text {th }}$ & $20 \mathrm{~N} / \mathrm{mm}$ & $I_{\text {th }}$ & $20 \mathrm{~N} / \mathrm{mm}$ \\
\hline 1 & $1.6 \mathrm{~A}$ & $55.06 \mathrm{~N}$ & $1.65 \mathrm{~A}$ & $52.70 \mathrm{~N}$ \\
2 & $1.6 \mathrm{~A}$ & $59.57 \mathrm{~N}$ & $1.65 \mathrm{~A}$ & $58.98 \mathrm{~N}$ \\
3 & $1.6 \mathrm{~A}$ & $54.53 \mathrm{~N}$ & $1.62 \mathrm{~A}$ & $47.47 \mathrm{~N}$ \\
\hline
\end{tabular}

\subsection{Pinch detection performance for $65 \mathrm{~N} / \mathrm{mm}$ test spring}

As stated in case of $20 \mathrm{~N} / \mathrm{mm}$ test spring, for each of the threshold method, the pinch detection performance using the current measurement are illustrated in Figure 9. When the pinch detection is made, the squeezing force is measured by the force sensor. Table 3 shows the squeezing force results for both methods.

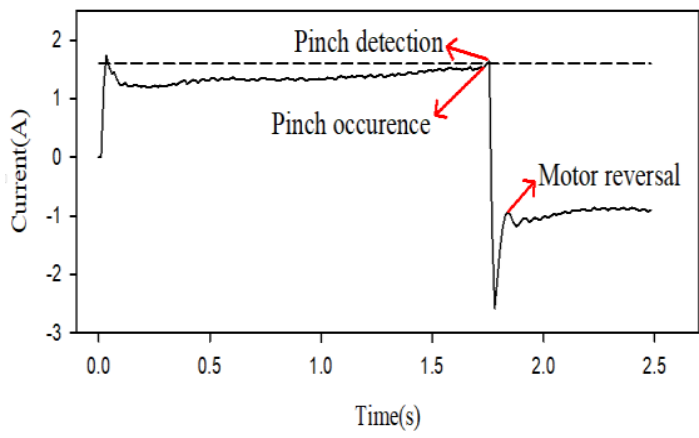

(a)

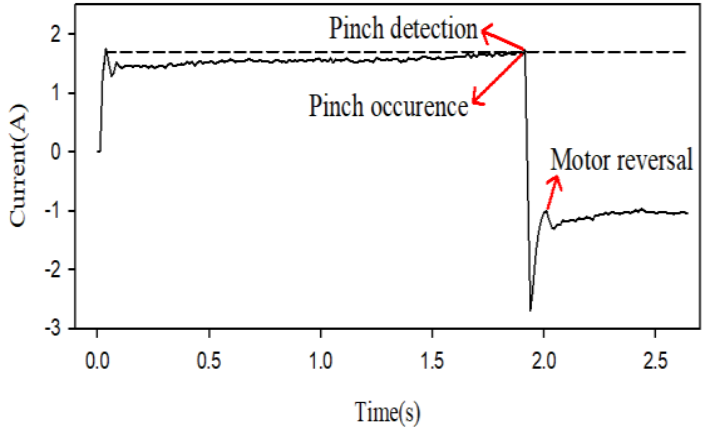

(b)

Figure 9. Pinch detection resuts using $65 \mathrm{~N} / \mathrm{mm}$ test spring (a) Manual threshold, (b) Automatic threshold 
Table 3. Squeezing Force Results for $65 \mathrm{~N} / \mathrm{mm}$ Test Spring

\begin{tabular}{ccccc}
\hline \multirow{2}{*}{ Test \# } & \multicolumn{2}{c}{ Manual threshold } & \multicolumn{2}{c}{ Automatic threshold } \\
& $I_{t h}$ & $65 \mathrm{~N} / \mathrm{mm}$ & $I_{\text {th }}$ & $65 \mathrm{~N} / \mathrm{mm}$ \\
\hline 1 & $1.6 \mathrm{~A}$ & $88.60 \mathrm{~N}$ & $1.69 \mathrm{~A}$ & $87.81 \mathrm{~N}$ \\
2 & $1.6 \mathrm{~A}$ & $85.87 \mathrm{~N}$ & $1.72 \mathrm{~A}$ & $87.57 \mathrm{~N}$ \\
3 & $1.6 \mathrm{~A}$ & $86.29 \mathrm{~N}$ & $1.69 \mathrm{~A}$ & $88.65 \mathrm{~N}$ \\
\hline
\end{tabular}

\subsection{Performance comparison and discussions}

As observed from Figure 8 and Figure 9, when the pinched object presents, the current rises until it reaches the threshold level. After reaching the threshold, the current does not continue to rise, but directly goes into negative value which means that the motor is working in reverse. It means that the pinch detection can be made quickly without any error with the current based anti-pinch system. Table 2 and Table 3 provide the test results for the performance comparison of the manual and automatic thresholds. For the manual threshold with a value 1.6A, the system can quickly detect the pinch condition without any error.

The squeezing forces of the anti-pinch system by using manual threshold are below $100 \mathrm{~N}$. For the automatic threshold method, there has been always little change to the threshold value. For the practical use, the power window system is likely to be affected by various disturbances that can lead to the electric current change as well. Also the heat from the DC motor leads to the current value drop. If the system is employing the manual threshold, the pinch condition detection will be delayed due to a larger gap between the threshold and the real current. But by using the automatic threshold, the threshold level will be adjusted depending on the system condition. Also the squeezing force of the anti-pinch system by using the automatic threshold is below $100 \mathrm{~N}$. From the test results for the anti-pinch system using manual and automatic thresholds, the performance of the both methods offers excellent results. The newly developed automatic threshold scheme for the current based anti-pinch system offers satisfactory results on the FMVSS 118 regulation. Finally, the low-cost technology has been employed to provide improved vehicle safety on power window while increasing convenience to the drivers or passengers.

\section{CONCLUSION}

In this research, the automatic threshold method for anti-pinch system has been proposed as a new method for pinch detection. By using the automatic threshold on power window system, the pinch condition can be detected as fast as the manual threshold performance. Also, the squeezing forces of this proposed method comply with the FMVSS 118 regulations $(<100 \mathrm{~N})$. The benefits of this method are that not only does the method give good performances, but also it makes the system more intelligent or smart. The automatic threshold can handle the disturbances that affected the system without making false pinch detection. Also this automatic threshold method on a current based anti-pinch system provides low cost of production and has a compact design which makes this system economically more competitive than other anti-pinch systems in the global market. Finally, this research discusses vehicle installation, test results, and performance comparisons for presented power window system.

\section{ACKNOWLEDGEMENT}

This work was supported by "Human Resources Program in Energy Technology" of the Korea Institute of Energy Technology Evaluation and Planning (KETEP), granted financial resource from the Ministry of Trade, Industry \& Energy, Republic of Korea. (No. 20164010200940).

\section{REFERENCES}

[1] T. Caussat and J. Everhart, "Safety with Convenience: Applying Low Cost Obstacle Detection Technology to Powered Closure Systems with Express Motion," SAE Tech. Pap., 2005.

[2] E. Kirby, "Child Safety via anti-trap proximity technology," SAE Tech. Pap., 2006.

[3] M. Sollmann, et al., "Anti Pinch Protection for Power Operated Features," SAE Tech. Pap., 2004.

[4] N. Brigitte and R. Herrmann, "Direct Sensor Solutions for Anti Pinch and Collision Avoidance for Motorized Closures," SAE Tech. Pap., 2009.

[5] K. S. Ho and J. S. Kwon, "Anti-Pinch System for Power Window by Using Hall Sensor," Korean Soc. Power Syst. Eng., vol. 14, no. 5, pp. 63-70, 2010.

[6] N. M. Yazdani and A. Y. Seqerloo, "Performance Comparison between Classic and Intelligent Methods for Position Control of DC Motor," Int. J. Electr. Comput. Eng., vol. 4, no. 3, pp. 366-371, 2014.

[7] J. S. Kwon, et al., "Safety mechanism design for automotive power windows using Hall sensor," Int. J. Veh. Saf., vol. 8, no. 2, pp. 129-143, 2015. 
[8] H. Lee and J. Park, "Practical pinch detection algorithm for low-cost anti-pinch window control system," in IEEE International Conference on Industrial Technology, pp. 995-1000, 2005.

[9] H. J. Lee, et al., "Robust Pinch Estimation and Detection Algorithm for Low-Cost Anti-Pinch Window Control Systems," Proc. IEEE Int. Conf. Ind. Technol., vol. 2005, pp. 269-274, 2005.

[10] J. H. Park, et al., "Real-time pinch detection algorithm: Robust to impulsive noise," Int. J. Control. Autom. Syst., vol. 7, no. 2, pp. 185-192, 2009.

[11] H. Alrawashdeh and A. Johnson, "The Kalman Filter Performance for Dynamic Change in System Parameters," IJECE Int. J. Electr. Comput. Eng., vol/issue: 3(6), pp. 713-723, 2013.

[12] B. D. Hoon, et al., "Anti-pinch Algorithm for Sunroof System Using Fuzzy Logic," in International Symposium on Advanced Intelligent Systems, pp. 1041-1046, 2005.

[13] W. K. Oleiwi, "Using the Fuzzy Logic to Find Optimal Centers of Clusters of K-means," Int. J. Electr. Comput. Eng., vol. 6, no. 6, pp. 3068, 2016.

[14] U.S. Department of Transportation, "National Highway Traffic Safety Administration Laboratory Test Procedure for FMVSS 118 Power-Operated Window, Partition , and Roof Panel Systems," 2006.

[15] "DIRECTIVE 2000/4/EC of the Eutopean Parliament and of the Council," Communities, Official J. of the European and. pp. 1-8, 2000.

[16] M. S. Widyan, et al., "Transient Analysis and Output Characteristics of DC Motors Fed by Photovoltaic Systems," Jordan J. Mech. Ind. Eng., vol. 4, no. 1, pp. 193-204, 2010.

\section{BIOGRAPHIES OF AUTHORS}
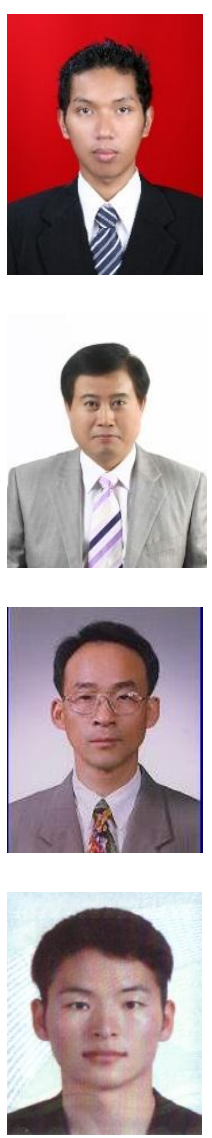

Wahyu Kunto Wibowo is with Department of Electrical Engineering, Universitas Pertamina, Jakarta, Indonesia. He received his M.Eng. and Ph.D. in Department of Interdisciplinary of Mechatronics Engineering from Pukyong National University in South Korea. His research interests are automotive safety system, motion control of AC and DC motor, design of robust control system, and electric propulsion system.

Seok-Kwon Jeong is a Professor in Department of Refrigeration and Air Conditioning Engineering at Pukyong National University, South Korea. He received his M.E. and Ph.D in Departement of Electrical and Information Engineering from Yokohama National University in Japan. His research intersts are automatic control of refrigeration and air conditioning system, motion control of AC motors, discrete system modeling, and electric propulsion system.

Sam-Sang You is a Professor in Division of Mechanical Engineering at Korea Maritime and Ocean University, South Korea. He earned the BS degree in the Marine Engineering at Pukyong National University, Korea in 1983, MS degree from the University of Wisconsin-Madison, USA in 1990, and PhD degree from Iowa State University, USA, in 1994, all in Mechanical Engineering. His research interests include system dynamics and robust controls, electric motor controls, and refrigeration system controls.

Jaemin Lee is with Department of Interdisciplinary of Mechatronics Engineering, PKNU Busan, 48547, Korea. He is interested in the part of advanced motion control by AC motors, application of PMSM, electric propulsion system, sensorless control, and sequential control by PLC. He is trying to apply this sensorless control to hybrid propulsion system for small ships which is receiving high attention because of eco-friendly environ 